\title{
Adiciones a la ficoflora marina de Venezuela. III. Ceramiales y Rhodymeniales (Rhodophyta)
}

Additions to the marine phycoflora of Venezuela. III. Ceramiales and Rhodymeniales (Rhodophyta)

\author{
Santiago Gómez ${ }^{1,4}$, Mayra García ${ }^{2} \&$ Nelson Gil ${ }^{3}$
}

\begin{abstract}
Resumen
Se realizó un inventario de las macroalgas de la zona submareal, entre 5 y $17 \mathrm{~m}$ de profundidad para las localidades de Puerto Maya, en el estado Aragua, Puerto Cruz, en el estado Vargas, Madrizquí y Boca de Cote, Parque Nacional Archipiélago Los Roques, Dependencias Federales. Como resultado se reportan cuatro nuevos registros de especies de algas rojas (Rhodophyta) para la costa de Venezuela: Apoglossum gregarium, Platysiphonia caribaea, Gloiocladia iyoensis y Botryocladia monoica. De las cuales, A. gregarium y G. iyoensis se registran también por primera vez en el Mar Caribe. Se establecen sus principales caracteres diagnósticos, resumidos como: hábito y dimensiones del talo, morfología de las estructuras reproductivas, presencia o ausencia de corticación y presencia o ausencia de células glandulares. Además se realizan comparaciones con sus especies morfológicamente cercanas.
\end{abstract}

Palabras clave: algas rojas, arrecifes coralinos, ficoflora marina, Venezuela, zona submareal.

\begin{abstract}
An inventory of the macroalgae of the subtidal zone from 5 to $17 \mathrm{~m}$ depth was made in the localities of Puerto Maya (Aragua state), Puerto Cruz (Vargas state), Madrizquí and Boca de Cote (Archipiélago Los Roques National Park). Four new records of red algal species (Rhodophyta) are reported for the Venezuelan coast: Apoglossum gregarium, Platysiphonia caribaea, Gloiocladia iyoensis and Botryocladia monoica. Two of them, A. gregarium and G. iyoensis are also reported for the first time for the Caribbean Sea. Their main diagnostic characteristics are established and summarized as follows: habit and dimensions of the thallus, morphology of the reproductive structures, presence or absence of cortical cells and gland cells. In addition, comparisons to morphologically related species are presented.
\end{abstract}

Key words: red algae, coral reefs, marine phycological flora, Venezuela, subtidal zone.

\section{Introducción}

El Mar Caribe es uno de los mares tropicales con mayor diversidad biológica del planeta y con una amplia variedad de formaciones costeras que favorecen el crecimiento de muchos organismos bénticos como las algas marinas (Bellorín 2003). Particularmente en la costa caribeña de Venezuela, se estima que existen alrededor de 600 especies de macroalgas (Ganesan 1989). Se han dado a conocer varios trabajos sobre la florística y distribución de estas especies, entre ellos destacan actualmente: Barrios et al. (2003), en el oriente del país, Ardito et al. (1995), Solé y Vera (1997) y García \& Gómez (2004) en el centro-occidente del país. Sin embargo la mayor parte de estos estudios se han limitado a la zona intermareal. Por esta razón, se han dedicado esfuerzos a estudiar la zona submareal de la costa continental central e insular venezolana por debajo de los 5 y hasta los $17 \mathrm{~m}$ de profundidad. El objetivo principal del presente trabajo es aportar la primera información florística que surge de esta investigación y contribuir a aumentar nuestro conocimiento de la ficoflora venezolana en ambientes hasta ahora escasamente estudiados.

\footnotetext{
${ }^{1}$ Universidad Central de Venezuela, Inst. Biología Experimental, Centro de Botánica Tropical, Apartado 20513, Caracas, Venezuela.

${ }^{2}$ Universidad Central de Venezuela, Fundación Instituto Botánico de Venezuela, Apartado 2156, Caracas, Venezuela.

${ }^{3}$ Universidad Pedagógica Experimental Libertador, Inst. Pedagógico de Miranda “José Manuel Siso Martínez”, Apartado 1073, Caracas, Venezuela..

${ }^{4}$ Autor para correspondencia: santiago.gomez@ciens.ucv.ve
} 


\section{Materiales y Métodos}

Los especímenes se colectaron mediante buceo autónomo, en arrecifes coralinos entre 5-17 m de profundidad, en cuatro localidades de la costa central de Venezuela: Puerto Maya, en el estado Aragua (10 $31^{\prime} 54^{\prime \prime} \mathrm{N}, 67^{\circ} 23$ '51'O), Puerto Cruz, en el estado Vargas (10³2'28'N, 67²0'39'O) y las localidades Madrizquí (11 ${ }^{\circ} 56^{\prime} 27.8^{\prime \prime} \mathrm{N}$, 6639'43.3”O) y Boca de Cote $\left(11^{\circ} 45^{\prime} 49.1^{\prime \prime} \mathrm{N}\right.$, 66²'06.6”O), Parque Nacional Archipiélago Los Roques, Dependencias Federales (Fig. 1).

Las muestras se preservaron en formaldehído al 4\% en agua de mar, luego se colorearon con una solución de safranina al 1\%, y se prepararon láminas semipermanentes utilizando una solución de $\mathrm{Karo}^{\circledR}$ al 30\%. Estas se depositaron en la colección de algas del Herbario Nacional de Venezuela (VEN). Las fotografías se tomaron utilizando un microscopio Nikon Eclipse E200 equipado con cámara digital modelo CoolPix-4500. Para la clasificación y nomenclatura se siguieron Wynne (2011) y Guiry \& Guiry (2012).

\section{Resultados y Discusión}

\section{Orden Ceramiales}

Familia Delesseriaceae Apoglossum gregarium (E.Y. Dawson) M.J. Wynne

Fig. 2a-f

Talo con porciones erectas y postradas, color rosado pálido, hasta $2 \mathrm{~mm}$ de alto, arraigado al sustrato mediante un sistema rizoidal. Porciones erectas formando láminas monostromáticas espatuladas, escasamente ramificadas, con un corto estipe terete y una costilla central conspicua, ápices emarginados. Porción basal de la lámina con ligera corticación rizoidal. Células axiales de 20-25 $\mu \mathrm{m}$ de diámetro, células pericentrales laterales, de 25-30 $\mu$ m de diámetro, sin división transversal. Esporangios tetraédricos, de 25-40 $\mu \mathrm{m}$ de diámetro, agrupados en soros pareados, dispuestos de manera ininterrumpida a lo largo de la costilla central, sobre ambas superficies de la lámina. Soros espermatangiales elipsoidales, constituidos por numerosas células madres, distribuidos de manera segregada sobre lámina y separados por falsas hileras de células estériles.

Material examinado: Estado Aragua, Puerto Maya, 3.VIII.2006, M. García et al., 1393, talo tetraspórico; 1393a, talo estéril; 1393b, talo masculino.

Presenta distribución mundial en el Golfo de California (EEUU), Islas Baleares, Francia, Italia, Mónaco, España (Europa), Bermuda (Islas del Atlántico), Islas Hawái (Islas del Pacífico), Sudáfrica (África) (Guiry \& Guiry 2012).

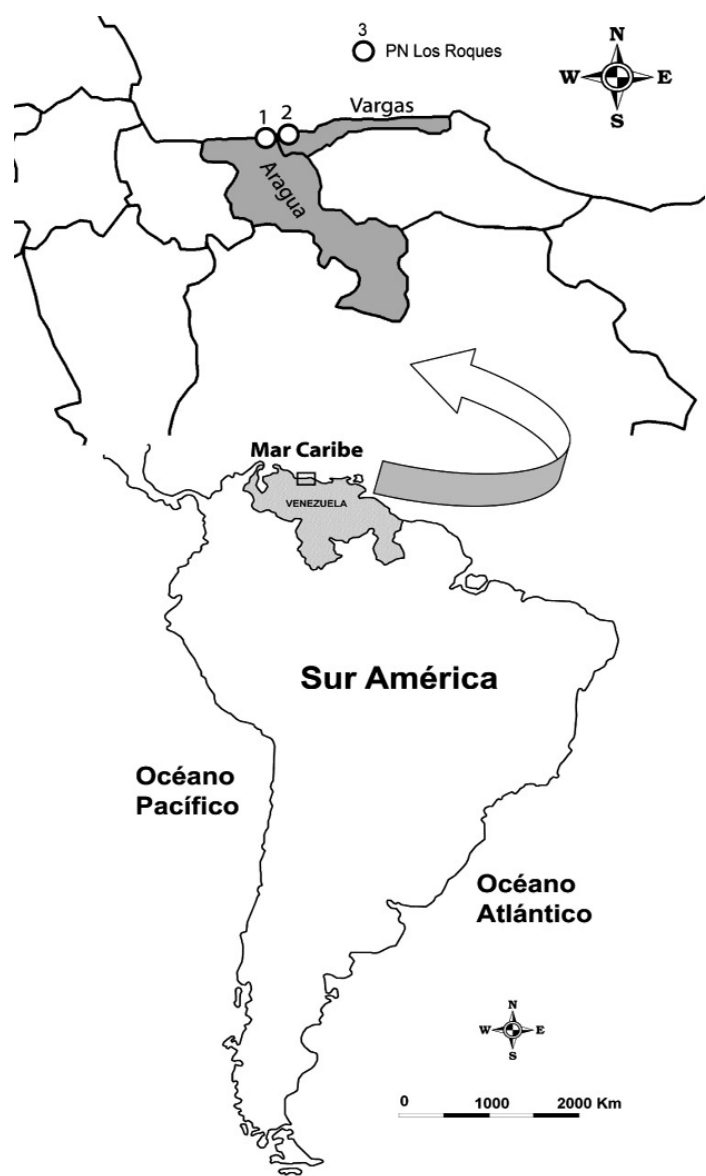

Figura 1- Mapa de la costa de Venezuela mostrando los sitios de muestreo.1. Puerto Maya, 2. Puerto Cruz, 3. Cayo Madrizquí y Boca de Cote.

Figure 1-Map of Venezuela coast showing the sampling sites. 1. Puerto Maya, 2. Puerto Cruz, 3. Madrizquí Cay and Boca de Cote.

Wynne (1985), describió la especie $A$. gregarium, como una combinación nueva a partir de Hypoglossum gregarium Dawson y Membranoptera spatulata Dawson, demostrando que corresponden al mismo taxón. Existen a nivel mundial siete especies aceptadas del género Apoglossum, de estas sólo dos son mencionadas para el océano Atlántico Occidental, A. gregarium y A. ruscifolium (Turner) J. Agardh (Wynne 2011), ambas muestran una cercana relación morfológica, pero esta última tiene un talo de tamaño mucho mayor (hasta $100 \mathrm{~mm}$ de largo), muy ramificado y además desarrolla soros espermatangiales en arreglo alargado, formando hileras paralelas, perpendiculares al eje central, lo cual difiere de A. gregarium cuyos soros espermatangiales son elipsoidales, distribuidos de manera segregada sobre lámina y separados por falsas hileras de células estériles (Schneider \& Searles 


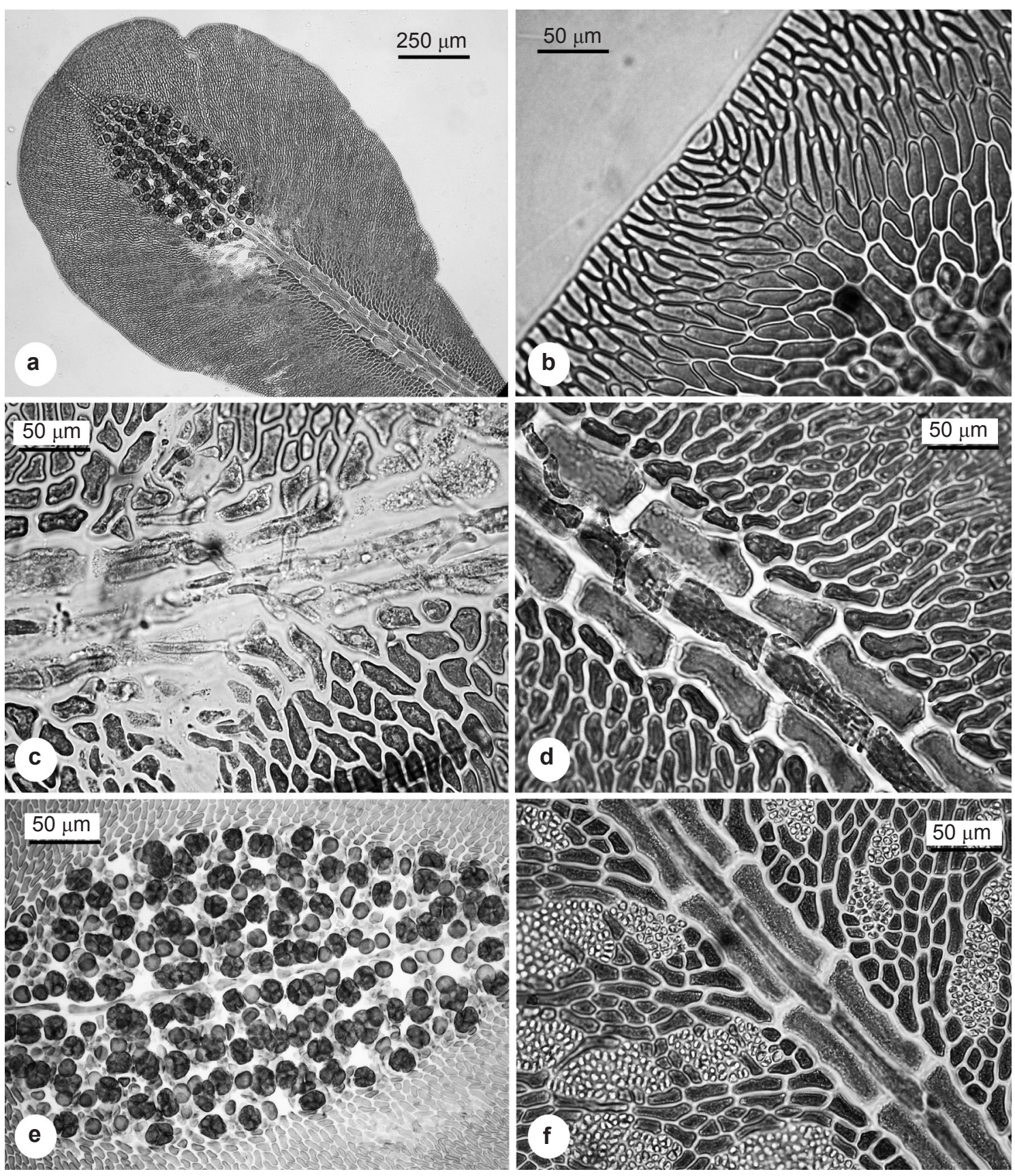

Figura 2 - a-f. Apoglossum gregarium - a. hábito del talo tetraspórico, b. detalle de la zona apical, c. detalle de la porción basal con ligera corticación rizoidal, d. detalle de la vena central conspicua, e. lámina con dos cámaras de tetrasporangios, f. lámina con paquete de espermatangios.

Figure 2 - a-f. Apoglossum gregarium - a. habit of the tetrasporic thallus, b. detail of the apical region, c. detail of the basal portion with light rizoidal cortication, d. detail of the conspicuous central vein, e. blade with two sori of tetrasporangia, f. blade with package of spermatangia.

1991; Maggs \& Hommersand 1993; Stegenga et al. 1997; Clavell \& Polo 2000).

Por otro lado existen dos especies australianas con similitudes morfológicas con $A$. gregarium, estas son A. spathulatum (Sonder) Womersley \& Shepley y
A. unguiculescens A.J.K. Millar, pero estas desarrollan ramas laterales a partir de la costilla central, mientras que en $A$. gregarium se originan desde el eje basal o a partir de fragmentos decumbentes (Wynne 1984; Littler \& Littler 2000; Horta \& Oliveira 2001). 
Orden Ceramiales

Familia Sarcomeniaceae

Platysiphonia caribaea D.L. Ballantine \& M.J. Wynne

Fig. 3a-d

Talo filamentoso, ecorticado, aplanado, con porciones erectas y postradas, color rosado rojizo, hasta $2 \mathrm{~mm}$ de alto, arraigado al sustrato mediante rizoides multicelulares, producidos desde las células más externas de los ejes postrados. Talo polisifonal, con cuatro células pericentrales por segmento. Células pericentrales laterales separadas por dos hileras paralelas de células acompañantes, a lo largo del eje. Tricoblastos ausentes. Ejes postrados de $150-160 \mu \mathrm{m}$ de diámetro y erectos de $90-100$ $\mu \mathrm{m}$ de diámetro; Ramas originándose a intervalos irregulares, desde el extremo proximal de la célula axial. Tetrasporangios tetraédricos de 40-50 $\mu \mathrm{m}$ de diámetro, separados por células pericentrales laterales. Material examinado: Estado Vargas, Puerto Cruz, 3.IX.2006, M. García et al. 1394 (VEN 299992), 1394 a (299991), talo estéril; Dependencias Federales, Parque Nacional Archipiélago Los Roques, Cayo Madrizquí, 31.VIII.2011, M. García \& S. Gómez 3165 (299990), talo tetraspórico.

Presenta distribución mundial en Bermuda, Islas Canarias, Islas de Cabo Verde (Islas del Atlántico), Bahamas (Islas del Caribe) (Guiry \& Guiry 2012).

Existen seis especies de Platysiphonia que carecen de corticación, distinguibles entre ellas por el patrón de división de las células flanqueantes en el estiquidio tetrasporangial (Silva \& Cleary 1954). $P$. caribaea es una de estas seis especies del género. $P$. clevelandii (Farlow) Papenfuss y $P$. delicata (Clemente) Cremades son las más cercanas a la especie en cuestión. $P$. delicata se diferencia ya que la división de las células flanqueantes de los ejes tetraspóricos es horizontal, mientras que en $P$. caribaea y $P$. clevelandii es vertical, sin embargo $P$. caribaea se distingue porque el par de células submarginales se divide periclinalmente, dando como resultado la producción de cuatro células submarginales, dos de cada lado de la lámina (Ballantine \& Wynne 1985).

Orden Rhodymeniales

Familia Faucheaceae

Gloiocladia iyoensis (Okamura) R.E. Norris

Figs. 3e-h,4 a-b

Talo erecto, complanado, mucilaginoso al tacto, color rosado rojizo, hasta 1,5-2,0 cm de alto, arraigado al sustrato mediante un disco de fijación. Ramificación predominantemente pinnada-dística, produciendo ocasionalmente ramas en un segundo plano, hacia las porciones terminales. Estructura multiaxial con células corticales pequeñas, en fascículos ramificados dicotómicamente, médula formada por células alargadas axialmente. Tetrasporangios ovoides, cruciados, de 25-50 $\mu \mathrm{m}$ de largo y de 25-30 $\mu \mathrm{m}$ de diámetro, originándose esparcidamente desde las células corticales, en las regiones distales de las ramas laterales. Soros espermatangiales superficiales, originándose de la división oblicua de las corticales más externas, ubicados hacia los ápices de las ramas laterales.

Material examinado: Estado Aragua, Puerto Maya, 3.III.2006, M. García \& S. Gómez. 1143a (VEN 299989), talo estéril, 1143b (VEN 299981), talo masculino; Dependencias Federales, Parque Nacional Archipiélago Los Roques, Boca de Cote, 30.VIII.2011, García y Gómez 3125 (VEN 299979), talo tetraspórico.

Presenta distribución mundial en Bermuda (Isla del Atlántico), Brasil, Sudáfrica, Japón, Corea, Australia, Nueva Zelanda, Estados Federales de Micronesia, Polinesia Francesa e Islas Hawái (Islas del Pacífico) (Guiry \& Guiry 2012).

Gloiocladia iyoensis muestra una estrecha relación con la especie G. atlantica (Searles) R.E. Norris, ambas especies desarrollan un patrón de ramificación pinnada-dística. Las diferencias se localizan a nivel de las dimensiones del talo, inferiores a $2 \mathrm{~cm}$ en el caso de G. iyoensis y de $2-5 \mathrm{~cm}$ en $G$. atlantica. Algunos autores como Norris (1991) y Schneider \& Lane (2007) consideran que se deben realizar estudios a fondo con estas dos especies, a nivel molecular, para dilucidar su presunta sinonimia.

\section{Orden Rhodymeniales}

Familia Rhodymeniaceae

Botryocladia monoica Schnetter.

Fig. $4 \mathrm{c}-\mathrm{h}$

Talo erecto, formado por un corto estipe y una vesícula terminal, color rosado pálido, hasta $4 \mathrm{~mm}$ de alto, arraigado al sustrato mediante un disco. Vesículas esféricas, huecas, de 1,5-2,0 $\mathrm{mm}$ de diámetro, ramificación escasa o ausente. Estructura constituida por una capa de células internas poligonales, de 20-30 $\mu \mathrm{m}$ de diámetro y de 70-90 $\mu \mathrm{m}$ de largo, y una capa incompleta de células corticales esféricas, de 15-20 $\mu \mathrm{m}$ de diámetro. Células glandulares piriformes, en grupos de 1-3, originándose desde las células medulares y proyectándose en la cavidad de la vesícula. Tetrasporangios ovoides, cruciados, de 20-25 $\mu \mathrm{m}$ de diámetro y de 30-35 $\mu \mathrm{m}$ de largo, originándose en la capa subcortical. Gametofitos monoicos, con cistocarpos esféricos, de 300-350 

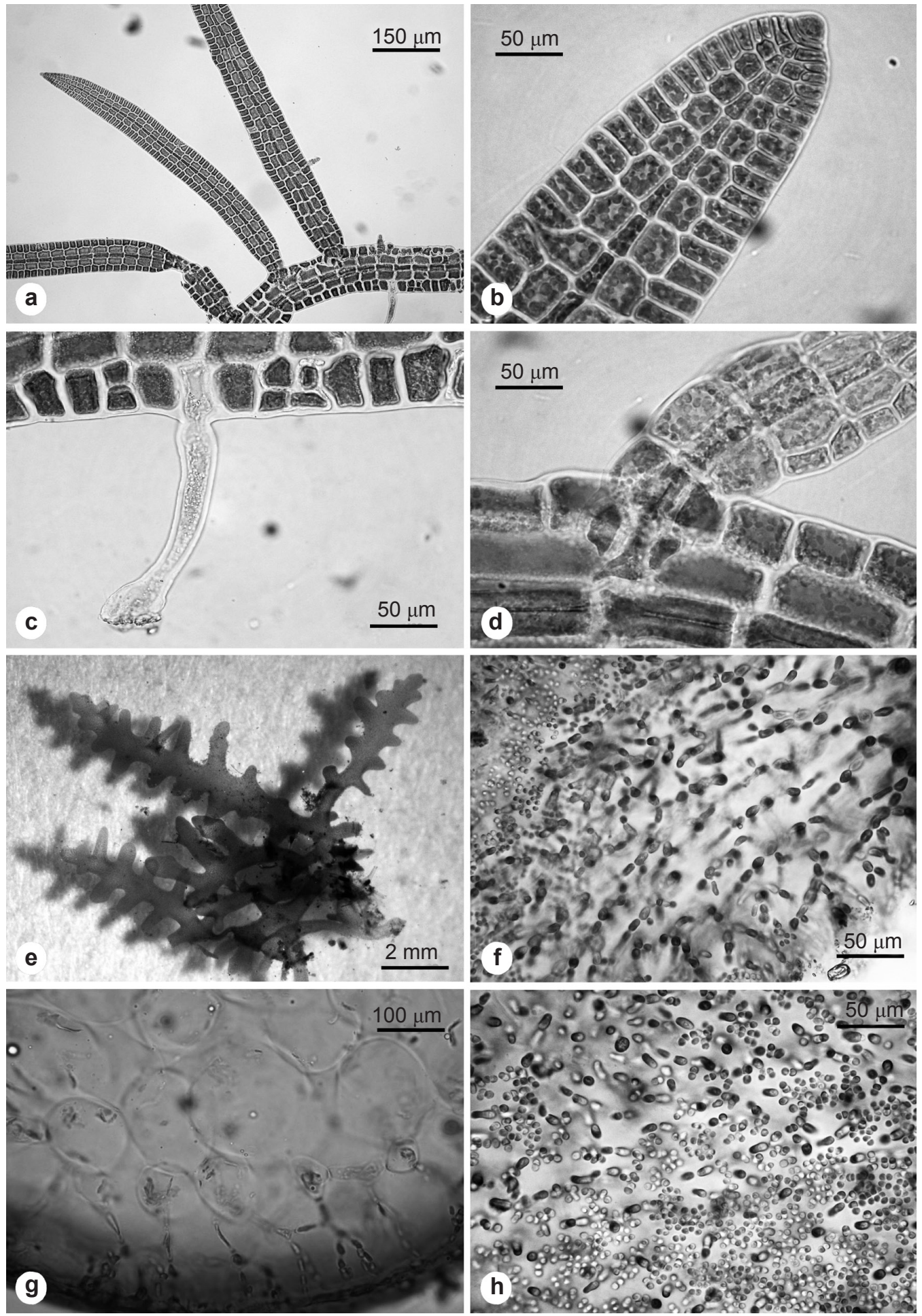

Figura 3 - a-d. Platysiphonia caribaea - a. hábito del talo estéril, b. detalle del ápice con célula apical, c. detalle de un rizoide, d. detalle de una rama joven procedente del extremo proximal de una célula axial. e-h. Gloiocladia iyoensis - e. hábito del talo estéril, f. vista superficial de fascículos de células corticales, g. corte transversal del talo, h. vista superficial de soros espermatangiales. Figure 3 - a-d. Platysiphonia caribaea - a. habit of the sterile thallus, b. detail of the apex with apical cell, c. detail of a rhizoid, d. Detail of a young branch coming from the proximal end of an axial cell. e-h. Gloiocladia iyoensis - e. habit of the sterile thallus, f. superficial view of fascicles of cortical cells, g. cross section of the thallus, $h$. superficial view of spermatangial sori. 

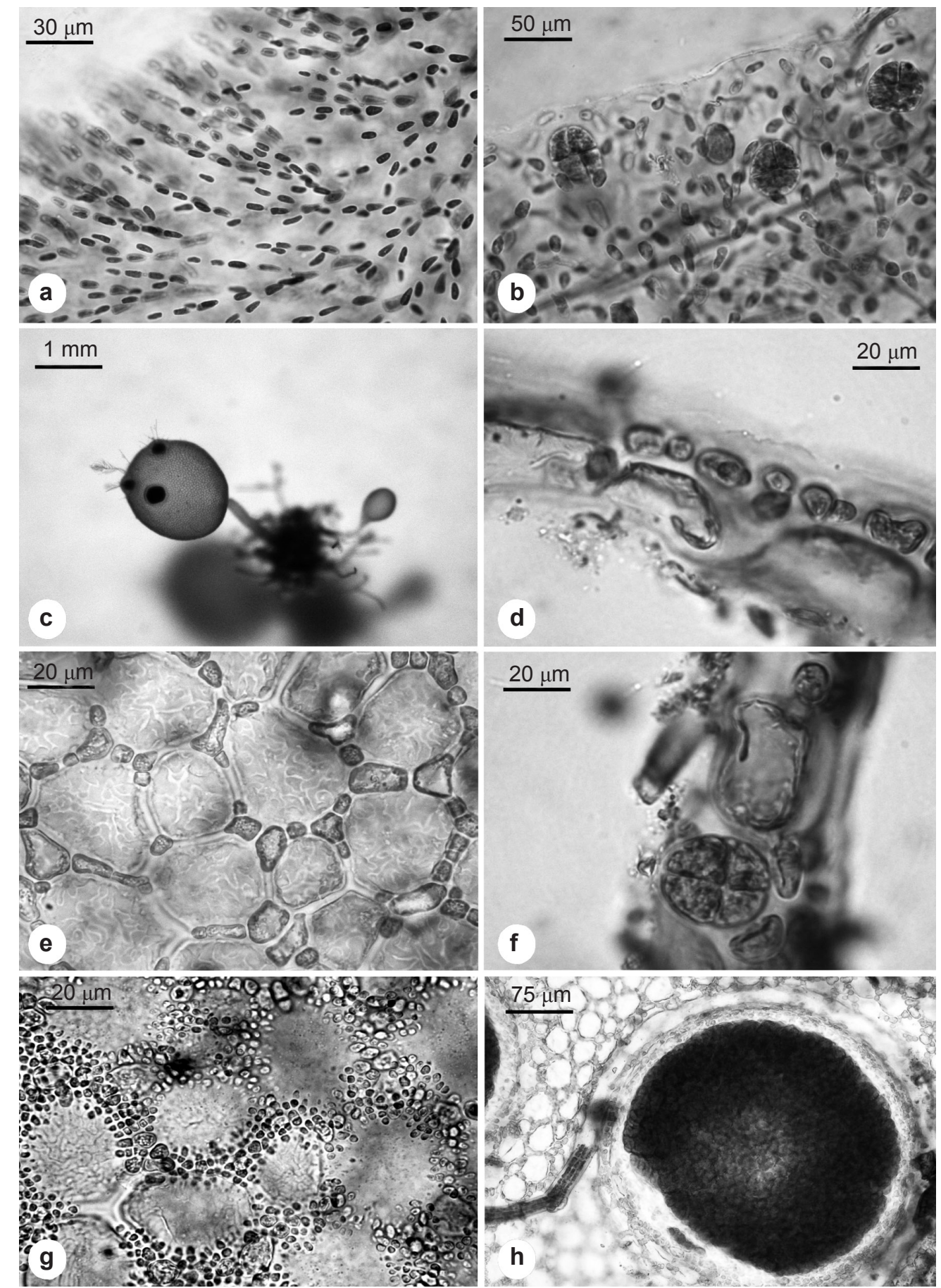

Figura 4 - a-b. Gloiocladia iyoensis - a. vista superficial de fascículos de células corticales, b. vista superficial de los tetrasporangios. c-h. Botryocladia monoica - c. hábito del talo cistocárpico, d. corte transversal de la vesícula mostrando células medulares y corticales, e. vista superficial de las paredes de la vesícula de un talo estéril, f. corte transversal de la vesícula mostrando tetrasporangio, g. vista superficial de las paredes de la vesícula con grupos de espermatangios, h. vista superficial de las paredes de la vesícula mostrando cistocarpo.

Figure 4 - a-b. Gloiocladia iyoensis - a. superficial view of fascicles of cortical cells, b. superficial view of the tetrasporangia. c-h. Botryocladia monoica $-\mathrm{c}$. habit of the cystocarpic thallus. d. cross section of the vesicle showing tetrasporangia, e. superficial view of the walls of the vesicle of a sterile thallus, f. cross section of the vesicle showing tetrasporangium, g. superficial view of the walls of the vesicle with groups of spermatangia, $h$. surface view of the walls of the vesicle showing a cystocarp. 
$\mu \mathrm{m}$ de diámetro, 1-3 por vesícula, células madre espermatangiales formadas desde las células corticales, agrupados alrededor de las células internas poligonales.

Material examinado: Estado Aragua, Puerto Maya, 30.III.2006, M. García et al. 1114 (VEN 299977), talo monoico (masculino y femenino); Estado Vargas, Puerto Cruz, 31.III.2006, 1158 (299974), talo tetraspórico.

Presenta distribución mundial en Texas (EEUU), Colombia (Mar Caribe), Puerto Rico (Isla del Caribe) (Guiry \& Guiry 2012).

Botryocladia monoica pertenece al grupo de especies de este género que poseen vesículas con una capa cortical incompleta y gametofitos monoicos, comparte estos caracteres con B. bahamensis Ballantine \& Aponte, B. ganesanii Aponte Díaz y $B$. wynnei Ballantine, pero estas tres especies exhiben tamaños superiores a $1 \mathrm{~cm}$, mientras que $B$. monoica no supera los $4 \mathrm{~mm}$. Por otra parte esta especie puede desarrollar hasta tres células glandulares, mientras que $B$. bahamensis presenta hasta ocho, $B$. ganesanii hasta 15 y B. wynnei hasta 17 (Schnetter 1978; Aponte-Díaz 1988).

Las cuatro especies citadas en este trabajo, todas pertenecientes a la división Rhodophyta, colectadas exclusivamente en aguas profundas, representan un valioso aporte al conocimiento de la biodiversidad de Venezuela, y se amplía el rango de distribución de estas algas en la geografía del Mar Caribe.

Dos de las especies que se adicionan son de distribución caribeña: Platysiphonia caribaea y Botryocladia monoica (Schnetter 1978; Schneider 2000), mientras que Apoglossum gregarium y Gloiocladia iyoensis han sido mencionadas en distintas regiones tropicales, sin embargo son especies poco comunes, siendo citadas por primera vez en el Mar Caribe.

Consideramos que el inventario ficoflorístico de la costa venezolana es todavía incompleto, conforme se realicen más colecciones en localidades poco estudiadas o inexploradas será posible adicionar especies a su ficoflora.

\section{Agradecimientos}

Los autores desean agradecer al Consejo de Desarrollo Científico y Humanístico de la Universidad Central de Venezuela, Proyecto PI 03-00-6422-2006, gracias a cuyo financiamiento parcial se pudo realizar la presente investigación. Igualmente al Dr. M. J. Wynne, por sus oportunas sugerencias para mejorar el manuscrito.

\section{Referencias}

Aponte-Díaz, M. 1988. Botryocladia ganesanii sp. nov. (Rhodophyta, Rhodymeniales) from the Caribbean coast of Venezuela. Cryptogamie, Algologie 9: 43-52.

Ardito, S.; Gómez, S. \& Vera, B. 1995. Estudio sistemático de las macroalgas bentónicas en la localidad de Taguao, Distrito Federal, Litoral Central, Venezuela. Acta Botanica Venezuelica 18: 53-66.

Ballantine, D.L. \& Wynne, M.J. 1985. Platysiphonia and Apoglossum (Delesseriaceae, Rhodophyta) in the tropical western Atlantic. Phycologia 24: 459-465.

Barrios, J.; Sant, S.; Méndez, E. \& Ruiz, L. 2003. Macroalgas asociadas a arrecifes coralinos en el Parque Nacional Mochima, Venezuela. Saber, Universidad de Oriente 15: 28-32.

Bellorín, A.M. 2003. Algas marinas bentónicas. In: Aguilera, M.; Azocar, A. \& González Jiménez, E. (eds). Biodiversidad en Venezuela. Vol. 1 Caracas, Fundación Polar y Ministerio de Ciencia y Tecnología. Pp. 94-103.

Clavell, A. \& Polo, L. 2000. Apoglossum gregarium (Delesseriaceae, Rhodophyta) en la Costa Brava (Gerona) y Baleares. Anales del Jardín Botánico de Madrid 57: 396-397.

Ganesan, E.K. 1989. A catalog of benthic marine algae and seagrasses of Venezuela. Fondo Editorial CONICIT, Caracas. 237p, 15 maps.

García, M. \& Gómez, S. 2004. Macroalgas bénticas marinas de la localidad Carmen de Uria, estado Vargas, Venezuela. Acta Botanica Venezuelica 27: 43-56.

Guiry, M.D. \& Guiry, G.M. 2012. AlgaeBase version 4.2. World-wide electronic publication, National University of Ireland, Galway. Disponible en $<$ http:// www.algaebase.org > . Acceso en 11 Dic 2011.

Horta, P.A. \& Oliveira, E.C. 2001. Some Delesseriaceae (Ceramiales, Rhodophyta) new to the southwestern Atlantic. Revista Brasileira de Botânica 24: 447-454.

Littler, D.S. \& Littler, M.M. 2000. Caribbean reef plants: an identification guide to the reef plants of the Caribbean, Bahamas, Florida and Gulf of Mexico. Offshore Graphics, Washington. 543p.

Maggs, C.A. \& Hommersand, M.H. 1993. Seaweeds of the British Isles. Vol. 1. Rhodophyta. Part 3A. Ceramiales. HMSO, London. 444p.

Norris, R.E. 1991. Some unusual marine red algae (Rhodophyta) from South Africa. Phycologia 30: 582-596.

Schneider, C.W. 2000. Notes on the marine algae of the Bermudas. 5. Some Delesseriaceae (Ceramiales, Rhodophyta), including the first record of Hypoglossum barbatum Okamura from the Atlantic Ocean. Botanica Marina 43: 455-466.

Schneider, C.W. \& Lane, C.E. 2007. Notes on the marine algae of Bermuda. 8. Further additions to the flora including Griffithsia aestivana sp. nov. (Ceramiaceae, Rhodophyta) and an update on 
the alien Cystoseira compressa (Sargassaceae, Heterokontophyta). Botanica Marina 50: 128-140.

Schneider, C.W. \& Searles, R.B. 1991. Seaweeds of the southeastern United States: Cape Hatteras to Cape Canaveral. Duke University Press, Durham, London. 553p.

Schnetter, R. 1978. Botryocladia monoica (Rhodymeniales, Rhodophyceae), a new species from the Caribbean coast of Colombia. Phycologia 17: $13-15$.

Silva, P.C. \& Cleary, A.P. 1954. The structure and reproduction of the red alga Platysiphonia. American Journal of Botany 41: 251-260.

Solé, M. \& Vera, B. 1997. Caracterización de las macroalgas bénticas en la región de Chirimena-
Punta Caimán, Edo. Miranda, Venezuela. Caribbean Journal of Science 33: 180-190.

Stegenga, H.; Bolton, J.J. \& Anderson, R.J. 1997. Seaweeds of the South African west coast. Contributions from the Bolus Herbarium 18: 1-655.

Wynne, M.J. 1984. The occurrence of Apoglossum and Delesseria (Ceramiales, Rhodophyta) in South Africa. South African Journal of Botany 3: 137-145.

Wynne, M.J. 1985. Taxonomic notes on some Delesseriaceae (Rhodophyta) occurring in southern California and Mexico. Bulletin of the Southern California Academy of Sciences 84: 164-171.

Wynne, M.J. 2011. A checklist of benthic marine algae of the tropical and subtropical Western Atlantic: third revision. Nova Hedwigia 140: 1-166. 